\title{
Similarity in the EDTA-soluble antigens of Clostridium chauvoei and C. septicum
}

\author{
Takafumi Hamaoka, ${ }^{1 *}$ Yasuyuki Mori, ${ }^{2}$ Nobuyuki Terakado ${ }^{2}$ and Shinichi Nakamura ${ }^{3}$ \\ ${ }^{1}$ Touhoku Branch Laboratory, National Institute of Animal Health, Shitinohe, Kamikita, Aomori 039-25, Japan \\ ${ }^{2}$ National Institute of Animal Health, Tsukuba Science City, Ibaraki 305, Japan \\ ${ }^{3}$ School of Medicine, Kanazawa University, Kanazawa 920, Japan
}

(Received 24 August 1992; revised 5 November 1992; accepted 18 November 1992)

\begin{abstract}
The EDTA-soluble antigens were prepared from whole cells of six strains of Clostridium chauvoei and five strains of $C$. septicum and were compared by sodium dodecyl sulphate-polyacrylamide gel electrophoresis (SDS-PAGE) and immunoblot analysis. SDS-PAGE profiles of the 11 strains were nearly identical, although there were slight variations in molecular mass in adjacent bands. In immunoblot analysis with two antisera against $C$. chauvoei and three against $C$. septicum, the antigens of all strains tested reacted with all five antisera and there were no differences in reactivities to the same antiserum between homologous and heterologous antigens. In an immunoblot reacted with a single antiserum, band patterns of 10 of the 11 strains were quite similar. After cross-absorption, antisera to both species lost most of their reactivities not only to heterologous antigens but also to homologous antigens. These results indicate that the two species share many common antigens and that there is a marked similarity in the antigenic properties of EDTA-soluble material.
\end{abstract}

\section{Introduction}

Clostridium chauvoei, which is the causative agent of blackleg in domestic animals, especially cattle and sheep, is closely related to Clostridium septicum, which causes gas gangrene in man and malignant oedema in animals. These two species have similar characteristics, except for fermentation of sucrose and salicin, and pathogenicity for man, rabbits and chickens (Willis, 1969; Smith \& Williams, 1984; Cato et al., 1986). Soluble cellular protein profiles on polyacrylamide gel electrophoresis are distinctive for each species but there are similarities (Cato et al., 1982).

Serological relationships between $C$. chauvoei and C. septicum have been reported by many workers. Moussa (1959) showed that the two species possessed distinguishable somatic and flagellar antigens but shared a common spore antigen. Using the fluorescent antibody technique, Batty \& Walker (1963) showed that there was no crossreaction between $C$. chauvoei and $C$. septicum. On the other hand, Weinberg \& Mihailesco (1929) showed that

*Author for correspondence. Tel. 017662 5115; fax 0176625117.

Abbreviation: PVDF, polyvinylidene difluoride. the two species were indistinguishable in complement fixation tests. More recently, Poxton \& Byrne (1984) showed that cross-reaction between $C$. chauvoei and $C$. septicum occurred in ELISA using EDTA extracts as antigens. Although cross-reactions in serological tests were reported, the properties of antigens shared by these species, apart from the spore antigen remain unclear.

This paper reports studies on the EDTA-soluble antigens of $C$. chauvoei and $C$. septicum with the objective of defining common antigens and extending the investigation of antigenic relationships between these two species.

\section{Methods}

Bacterial strains. C. chauvoei strains ATCC 10092, ATCC 19399, ATCC 11956, ATCC 11957, ATCC 11958 and Okinawa were used. Strain Okinawa, which is used for blackleg vaccination in Japan, is maintained in our laboratory. The other strains were from the American Type Culture Collection, Rockville, MD, USA. ATCC 10092 is the type strain of $C$. chavvoei. $C$. septicum strains ATCC 12464, NCTC 286, NCTC 551, NCTC 282 and NCTC 504 were used. ATCC 12464, which is the type strain, was from the American Type Culture Collection. The other strains were from the National Collection of Type Cultures, Central Public Health Laboratory, London, UK.

Growth conditions. All strains were cultured in BTHPYG medium ( $\mathrm{pH} 7 \cdot 2$ ), which was modified from THPYG medium (Nakamura et al., 
1979), and contained $0.5 \%(\mathrm{w} / \mathrm{v})$ trypticase (BBL), $0.5 \%(\mathrm{w} / \mathrm{v})$ heart infusion broth (Difco), $1.0 \%(\mathrm{w} / \mathrm{v})$ polypeptone (Daigo), $1.0 \%(\mathrm{w} / \mathrm{v})$ yeast extract (BBL), $1.0 \%(\mathrm{w} / \mathrm{v})$ glucose, $0.5 \%(\mathrm{w} / \mathrm{v}) \mathrm{NaCl}, 0.02 \%$ (w/v) $\mathrm{KCl}, 0.115 \%(\mathrm{w} / \mathrm{v}) \mathrm{Na}_{2} \mathrm{HPO}_{4}, 0.02 \%(\mathrm{w} / \mathrm{v}) \mathrm{KH}_{2} \mathrm{PO}_{4}, 0.05 \%$ $(\mathrm{w} / \mathrm{v}) \mathrm{L}$-cysteine. $\mathrm{A} 1.0 \mathrm{ml}$ volume of stationary-phase bacterial culture in cooked meat medium (Difco) was inoculated into $15 \mathrm{ml}$ of BTHPYG medium. Cultures were incubated aerobically, unshaken, at $37^{\circ} \mathrm{C}$. At mid-exponential phase, $1.0 \mathrm{ml}$ volumes of the cultures were inoculated into the same medium again. These subcultures were repeated three times. This reduced the incubation time for yielding mid-exponential phase cultures. Finally, $15 \mathrm{ml}$ of mid-exponential-phase culture was inoculated into $200 \mathrm{ml}$ fresh medium, and cultures were statically incubated aerobically for 4 or $5 \mathrm{~h}$ at $37^{\circ} \mathrm{C}$. The bacterial cells were then harvested by centrifugation $(10000 \mathrm{~g}, 15 \mathrm{~min})$. Sporulation was negligible under these conditions.

EDTA-soluble antigens. The EDTA-soluble antigens were prepared from whole cells by the method of Poxton \& Byrne (1981) and Poxton (1984). Protein concentrations of the antigens were measured by the Bio-Rad protein assay kit or by absorbance at $280 \mathrm{~nm}$.

Preparation of antisera and cross-absorption treatment of sera. Antisera against each of the EDTA-soluble antigens from $C$. chauvoei ATCC 10092 and ATCC 11958, and from C. septicum NCTC 286, NCTC 282 and NCTC 504, were prepared in Japanese White rabbits. The antigens in Freund's complete adjuvant (Iatron) were injected subcutaneously into rabbits six times at $10 \mathrm{~d}$ intervals. Crossabsorption treatments of antisera were carried out as follows. Undiluted antiserum was mixed with an equal volume of heterologous EDTA-soluble antigen. The mixture was kept standing overnight at $4{ }^{\circ} \mathrm{C}$. After that, a further equal volume of the antigen was added and the mixture kept standing overnight again at $4{ }^{\circ} \mathrm{C}$. These treatments were repeated until the antiserum lost reactivity to the antigen used for absorption. Protein concentration of the antigen used in absorption treatment was approximately $2 \mathrm{mg} \mathrm{ml}^{-1}$.

SDS-PAGE. This was performed on $12 \%(\mathrm{w} / \mathrm{v})$ slab gels with the buffer system of Laemmli (1970). Approximately $10 \mu \mathrm{g}$ of protein was run in each track. Protein bands on the gels were stained with $0.25 \%$ (w/v) Coomassie brilliant blue R-250 in 50\% (v/v) methanol, $9 \%(v / v)$ acetic acid.

Immunoblotting. Immunoblots were prepared as follows; Electroblot transfer was performed by the method of Towbin et al. (1979). Antigens on SDS-PAGE gel were transferred to polyvinylidene difluoride (PVDF) membrane (Immobilon; Millipore) in a horizontal transblotting chamber (Atto) containing $20 \%(\mathrm{v} / \mathrm{v})$ methanol, $20 \mathrm{~mm}$-Tris, and $150 \mathrm{~mm}$-glycine buffer (pH 8.3) for $45 \mathrm{~min}$ at $200 \mathrm{~mA}$ current. Protein-free sites were saturated by overnight incubation at $4{ }^{\circ} \mathrm{C}$ in Tris-buffered saline (10 mM-Tris, $0.9 \%$, w/v, NaCl, pH 7.4; TBS) containing $3 \%(\mathrm{w} / \mathrm{v})$ bovine serum albumin (Sigma). The PVDF membrane was incubated at room temperature for $2 \mathrm{~h}$ with adequate dilutions [in $1 \%(\mathrm{w} / \mathrm{v})$ skim milk TBS] of rabbit antiserum raised against EDTA-soluble antigen. After three washes (10 min each) in $0.02 \%(\mathrm{v} / \mathrm{v})$ Tween 20 saline, it was incubated for $1 \mathrm{~h}$ with goat antirabbit IgG conjugated with horseradish peroxidase (Cappel, Organon Teknika) diluted 1:5000 with $1 \%$ skim milk TBS. After three washes (10 min each) in Tween saline, it was placed into substrate solution $60 \mathrm{mg}$ (4-chloro-1-naphthol, $20 \mathrm{ml}$ methanol, $6 \mu \mathrm{l} \mathrm{H} \mathrm{O}_{2}$, and $100 \mathrm{ml}$ TBS) for colour development. Colour developed within 5-15 min. The reaction was stopped by washing with distilled water. All the steps mentioned above were done with gentle agitation throughout.

Protein bands on the membrane were detected by staining with $0 \cdot 1 \%$ (w/v) Amido black 10B in 50\% (v/v) methanol, $10 \%$ (v/v) acetic acid or Colloidal Gold Total Protein Stain (Bio-Rad) with Gold Enhancement kit (Bio-Rad). Pre-stained SDS-PAGE standards (low range; Bio-Rad) were used for estimation of the molecular mass of protein bands.

\section{Results}

$S D S-P A G E$ profiles of EDTA-soluble antigens of $C$. chauvoei and $C$. septicum

The EDTA-soluble antigens prepared from C. chauvoei Okinawa, ATCC 10092, ATCC 19399, ATCC 11956, ATCC 11957 and ATCC 11958, and from C. septicum ATCC 12464, NCTC 286, NCTC 551, NCTC 282 and NCTC 504, were compared on an SDS-PAGE gel and an electroblot transfer membrane. The gel was stained with Coomassie blue, and the membrane was stained by Amido black. The electroblot transfer of protein bands from gel to PVDF membrane was effective in all of the range $10-110 \mathrm{kDa}$. Protein band profiles on the gel and the membrane were quite similar. Band profiles of all the antigens examined were complex and quite similar except for that of $C$. septicum ATCC 12464. There were no significant differences in the band profiles of the two species (Fig. 1). No species-specific band was found. However, the molecular masses of adjacent major bands found in $C$. chauvoei and $C$. septicum were slightly different (Fig. $1 a$, indicated by arrowheads). Weak or missing bands in some strains were noted, but these variations were not specific for each species (Fig. $1 a$, indicated by arrows). The antigen of $C$. septicum ATCC 12464 (lane 7) consisted of only one major band of molecular mass approximately $43 \mathrm{kDa}$ and some minor bands.

\section{Immunoblot analysis of EDTA-soluble antigens of C. chauvoei and C. septicum}

Immunoblots of the EDTA-soluble antigens of C. chauvoei Okinawa, ATCC 10092, ATCC 19399, ATCC 11956, ATCC 11957 and ATCC 11958, and of C. septicum ATCC 12464, NCTC 286, NCTC 551, NCTC 282 and NCTC 504, reacted with antisera against the antigens of $C$. chauvoei ATCC 10092 and ATCC 11958, and of C. septicum NCTC 286, NCTC 282 and NCTC 504, were examined. Each antiserum reacted in the immunoblots to all antigens, not only those which were homologous but also those which were heterologous antigens.

In an immunoblot reacted with the antiserum raised against $C$. chauvoei ATCC 10092 antigen (Fig. 2), several bands which were found at $29,36,42-43,50$ and $88 \mathrm{kDa}$ were observed in the $C$. septicum strains (lanes 7-11). Although these visible bands in $C$. septicum indicated their common antigenicities to $C$. chauvoei, the molecular masses of some bands were slightly different from their corresponding bands in C. chauvoei. A band in $C$. 

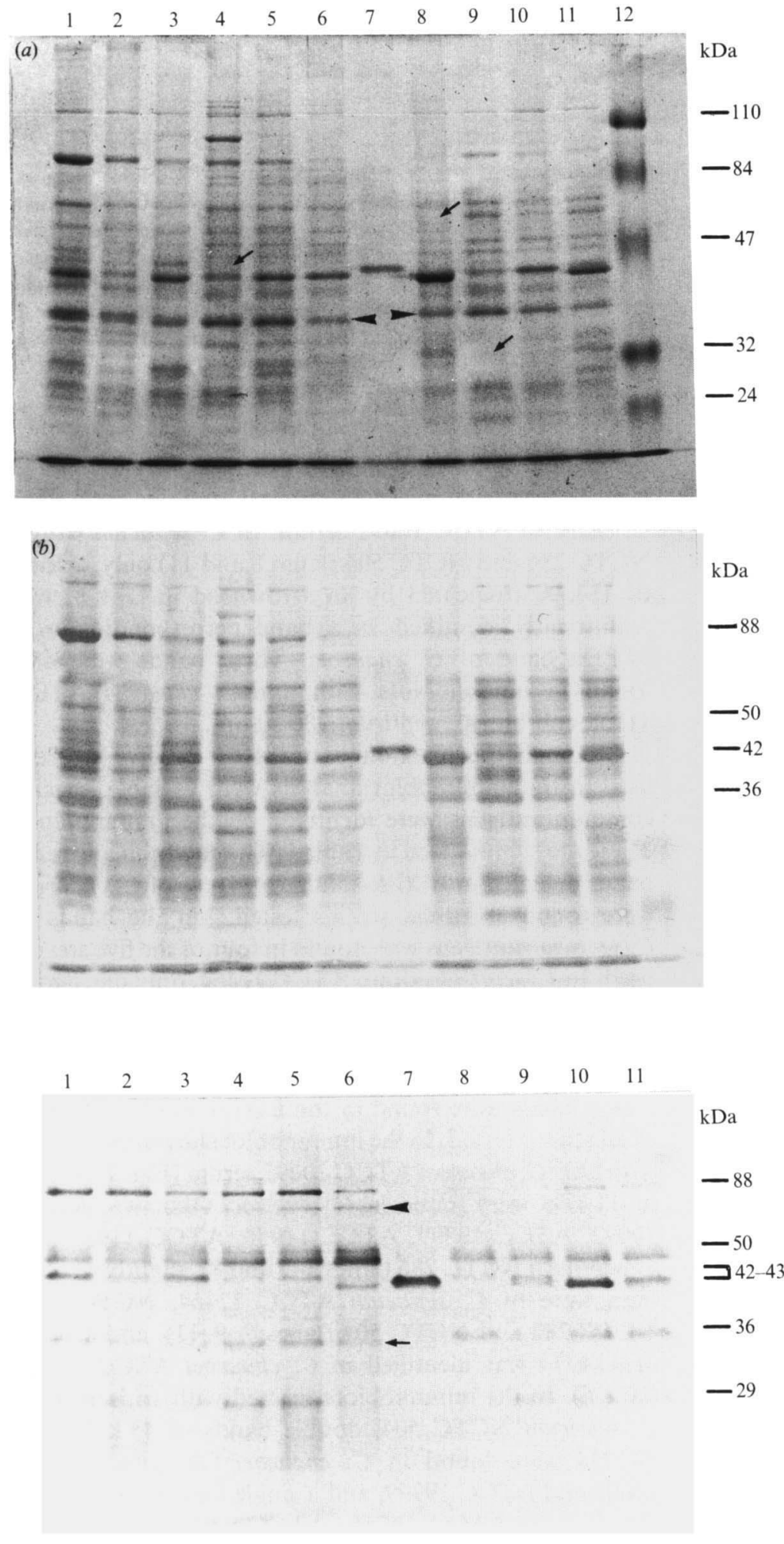

Fig. 1. SDS-PAGE and corresponding electroblot transfers of EDTA-soluble antigens from strains of C. chauvoei and C. septicum. Lane 1, C. chauvoei Okinawa; lane 2, C. chauvoei ATCC 10092; lane 3, C.chauvoeiATCC 19399; lane 4,C. chawvoei ATCC 11956; lane 5, C. chauvoei ATCC 11957; lane 6, C.chauvoei ATCC11958; lane 7,C.septicumATCC 12464; lane 8,C. septicum NCTC 286; lane 9, C. septicum NCTC 551 ; lane $10, C$. septicum NCTC 282; lane 11, C. septicum NCTC 504; lane 12, molecular mass markers. (a) Gel stained with Coomassie brilliant blue. (b) Electroblot transfer membrane of (a) stained with Amido black. Arrowheads in lanes 6 and 8 in $(a)$ indicate variation in molecular mass of corresponding bands in C. chauvoei and C. septicum. Arrows indicate weak or missing bands in some strains.
Fig. 2. Immunoblot of EDTA-soluble antigens from strains of $C$. chauvoei and $C$. septicum reacted with an antiserum raised against the antigen of $C$. chauvoei ATCC 10092. The arrangement of strains is the same as in Fig. 1. An arrowhead indicates the $78 \mathrm{kDa}$ antigenic band. The arrow indicates the $35 \mathrm{kDa}$ antigen. chauvoei corresponding to a band of $36 \mathrm{kDa}$ in $C$. septicum was found at $35 \mathrm{kDa}$ (Fig. 2, arrow, lane 6). Moreover, interestingly, molecular mass variation was observed in the range $42-43 \mathrm{kDa}$ among all the 11 strains tested. On the other hand, a band of $78 \mathrm{kDa}$ was found in only C. chauvoei (lanes $1-6$, indicated by an arrowhead 


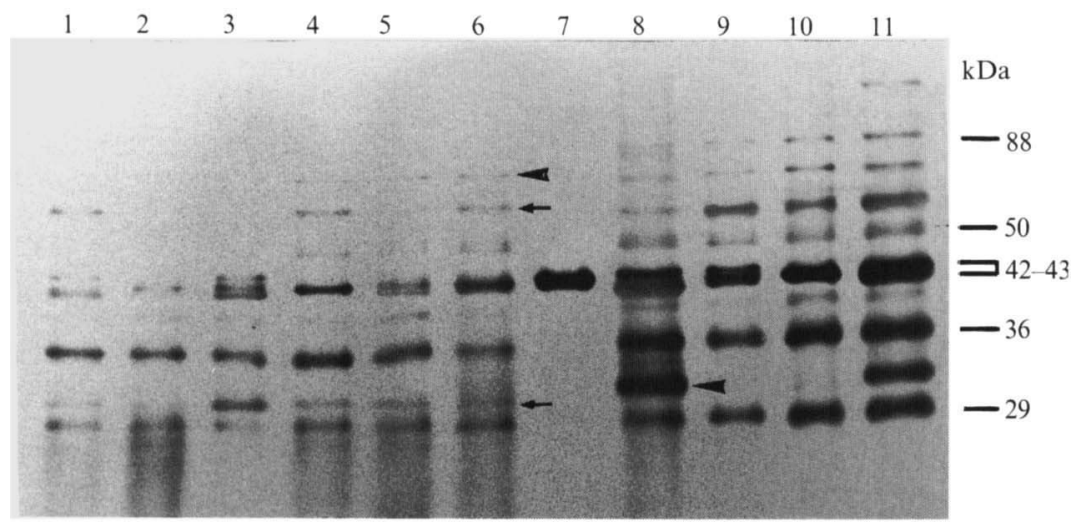

Fig. 3. Immunoblot of EDTA-soluble antigens from strains of $C$. chavoei and $C$. septicum reacted with an antiserum raised against the antigen of $C$. septicum NCTC 504. The arrangement of strains is the same as in Fig. 1. Arrowheads indicate the $78 \mathrm{kDa}$ antigen in lane 6 and the $32 \mathrm{kDa}$ antigen in lane 8 . Arrows indicate the 66 and $31 \mathrm{kDa}$ antigens.
Table 1. Summary of major antigens of $C$. chauvoei and C. septicum detected on immunoblots of EDTA-soluble antigens

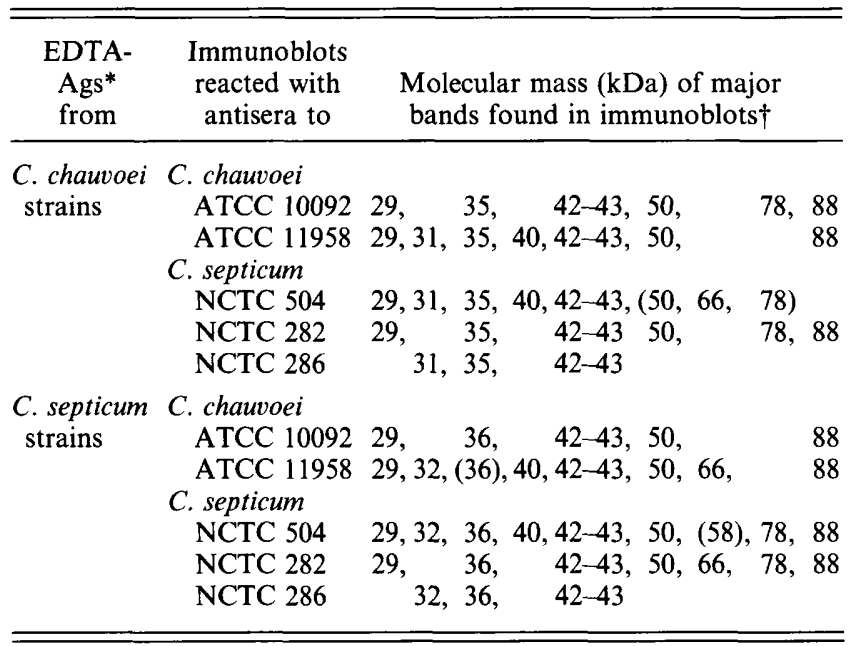

* EDTA-Ags, EDTA-soluble antigens.

+ Values in parentheses indicate the molecular masses of weak bands.

in lane 6). However, because a similar band was revealed by reaction with the antiserum against $C$. septicum NCTC 504 antigen (Fig. 3, indicated by an arrowhead in lane 6) this band cannot be recognized as a specific antigen for $C$. chauvoei. A similar profile was observed on an immunoblot reacted with the antiserum against C. septicum NCTC 282 antigen.

The profiles in an immunoblot reacted with the antiserum against antigen of $C$. septicum NCTC 504 (Fig. 3) were similar to those in the immunoblot using anti-C. chauvoei ATCC 10092 serum (Fig. 2), with the exception of a few bands. Although reactions were weak in the higher molecular mass region, visible bands indicating their common antigenicities to $C$. septicum were observed at 29, 31, 35, 40, 42-43, 50, 66 and $78 \mathrm{kDa}$ in C. chauvoei strains (Fig. 3, lanes 1-6). Among them, the bands of 31 and $66 \mathrm{kDa}$ (indicated by arrows in lane 6) were not found in the immunoblot with antiC. chauvoei ATCC 10092 serum. In C. septicum strains NCTC 286 and NCTC 504 (lanes 8 and 11) only, a band of $32 \mathrm{kDa}$ (indicated by an arrowhead in lane 8) was additionally identified as a band corresponding to a $31 \mathrm{kDa}$ band of $C$. chauvoei. These bands were also found in immunoblots with anti-C. chauvoei ATCC 11958 and anti-C. septicum 286 serum.

Although the reaction patterns of the five antisera used in the immunoblot analysis were variable, several common antigens were identified on the immunoblots. These are summarized in Table 1. The common antigenic bands detected in EDTA-soluble antigens of $C$. chauvoei were found in all six strains tested, but the bands in C. septicum antigens were found in four of the five strains used. In C. septicum strain ATCC 12464, only one major band of around $43 \mathrm{kDa}$ appeared characteristically in all of the immunoblots. Although strain-specific molecular mass variation was observed in the range $42-43 \mathrm{kDa}$, major bands were found in the EDTA-soluble antigens of all strains tested. In the immunoblot showing reactions with anti-C. chauvoei ATCC 10092 serum (Fig. 2), bands of $43 \mathrm{kDa}$ were found in C. chauvoei Okinawa, ATCC 10092, ATCC 19399, ATCC 11956, ATCC 11957 and C. septicum NCTC 286 (lanes 1-5 and 8), slightly smaller ones were in C. septicum ATCC 12464, NCTC 551, NCTC 282 and NCTC 504 (lanes 7, 9-11), and a band of $42 \mathrm{kDa}$ was identified in C. chauvoei ATCC 11958 (lane 6). In the immunoblots reacted with antiserum to C. septicum NCTC 504, double bands of $43 \mathrm{kDa}$ and $42 \mathrm{kDa}$ were found in $C$. chauvoei Okinawa, ATCC 10092 and ATCC 19399, and a single band of $42 \mathrm{kDa}$ in C. chauvoei ATCC 11956, ATCC 11957 and ATCC 11958 (Fig. 3). The bands of $C$. septicum in the range 42-43 kDa were similar to those obtained with antiC. chauvoei ATCC 10092 serum. A band found at $50 \mathrm{kDa}$ in $C$. septicum was characteristically broad, but the bands in C. chauvoei were doubled. 


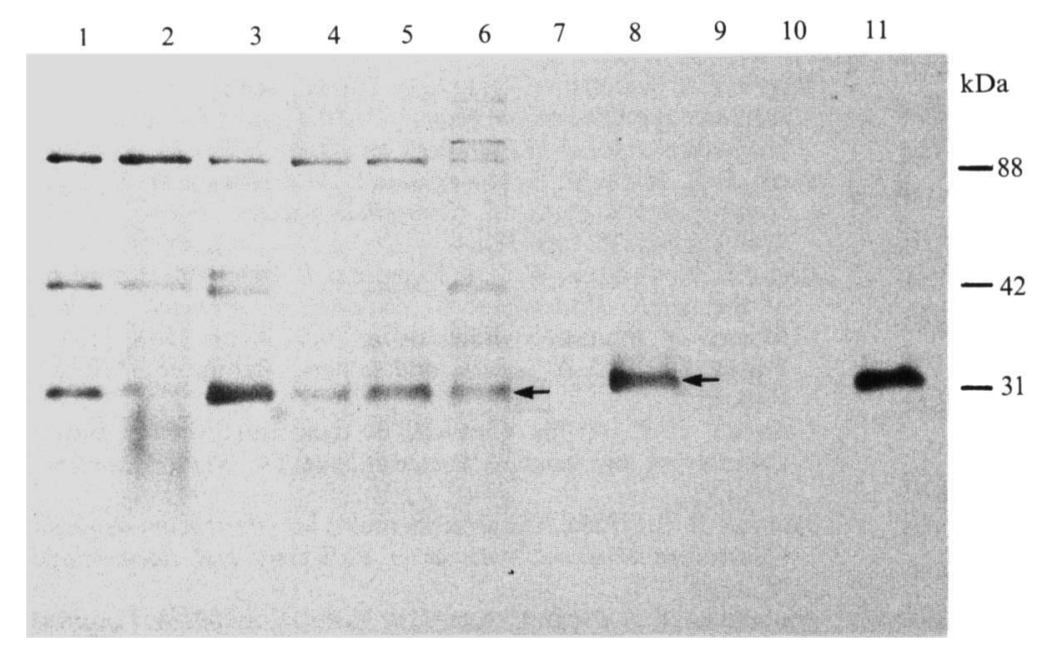

Fig. 4. Immunoblots of EDTA-soluble antigens from strains of $C$. chauvoei and $C$. septicum reacted with antiserum to the antigen of $C$. chauvoei ATCC 11958 which was absorbed with the antigen of $C$. septicum NCTC 282. The arrangement of strains is the same as in Fig. 1. Arrows in lanes 6 and 8 indicate 31 and $32 \mathrm{kDa}$ antigens.
In each antigen of $C$. chauvoei and C. septicum, there was no band detected by staining only with their homologous antiserum.

\section{Cross-absorption test for analysis of common antigens}

Immunoblots were prepared using antisera against C. chauvoei ATCC 11958 antigen absorbed with each of the EDTA-soluble antigens from C. septicum NCTC 282 and NCTC 504, and with an antiserum against C. septicum NCTC 282 antigen absorbed with $C$. chauvoei ATCC 11958 antigen. The antiserum against C. chauvoei ATCC 11958 antigen absorbed with $C$. septicum NCTC 282 antigen lost nearly all reactivity with the exception of three bands of 88,42 and $31 \mathrm{kDa}$ in $C$. chauvoei and a band of $32 \mathrm{kDa}$ in C. septicum NCTC 286 and NCTC 504 (Fig. 4). The same antiserum absorbed with $C$. septicum NCTC 504 antigen which included a $32 \mathrm{kDa}$ antigen lost further reactivity to bands of $31 \mathrm{kDa}$ in $C$. chauvoei and of $32 \mathrm{kDa}$ in C. septicum NCTC 286 and NCTC 504. The antiserum against C. septicum NCTC 282 antigen absorbed with $C$. chauvoei ATCC 11958 antigen reacted only with bands of $42 \mathrm{kDa}$ in C. septicum NCTC 282 and ATCC 12464. The band of C. septicum ATCC 12464 was much weaker than that of $C$. septicum NCTC 282.

\section{Discussion}

The results obtained with the SDS-PAGE analysis indicate that although the staining density or molecular mass of some bands were slightly different in each strain examined, EDTA-soluble protein profiles of $C$. chauvoei and $C$. septicum closely resembled each other. There were no distinct differences to discriminate these related species. Cato et al. (1986) have also reported that electrophoretic patterns of soluble cellular proteins of
C. chauvoei and C. septicum were extremely similar but a marked difference, found in the higher-molecular-mass region, clearly separated them. Our results in this paper are not in agreement with that finding: we have examined EDTA-soluble proteins, which comprise a limited fraction of soluble cellular protein, while they examined the whole soluble protein fraction. The electrophoretic procedure we used is also different from theirs.

The results obtained by immunoblot analysis indicate conclusively that $C$. chauvoei shares many antigens with $C$. septicum and that these common antigens are specifically solubilized from whole cells by EDTA treatment. Species-specific antigens of $C$. chauvoei and C. septicum were not found in the EDTA-soluble antigens. Although a few species-specific bands were found in immunoblots using antisera absorbed with heterologous antigen, the bands were weaker than those stained by unabsorbed sera. Therefore, these bands should not be regarded as reliable species-specific antigens. Poxton \& Byrne (1984) demonstrated that cross-reaction between $C$. chauvoei and $C$. septicum occurred in ELISA in which EDTA extract was used as ELISA-antigen. Our study provides corroborative evidence for antigenic similarity between the EDTAsoluble antigens of $C$. chauvoei and $C$. septicum.

The five antisera used in this study showed different reactivities in immunoblots. In each immunoblot, however, there were marked similarities in reaction patterns between homologous and heterologous antigens. Therefore, variety among immunoblot patterns of the five antisera used should be attributed to quantitative variation of antigenic components included in each antigen rather than to qualitative differences.

There were slight differences in molecular mass of corresponding bands in $C$. chauvoei and C. septicum, e.g. bands of $35 \mathrm{kDa}$ appeared in all $C$. chawvoei strains, but corresponding bands in $C$. septicum antigens appeared at 
$36 \mathrm{kDa}$ (Figs 2 and 3). Additionally, bands at $31 \mathrm{kDa}$ were visible in $C$. chauvoei but corresponding bands of C. septicum were found at $32 \mathrm{kDa}$ in two strains (lanes 8 and 11 in Fig. 3). These bands of 31 and $32 \mathrm{kDa}$ simultaneously disappeared in cross-absorption tests. These results indicate that while the molecular masses of these corresponding bands are slightly different, their antigenicities are very similar. Although these slight differences were not sufficient to characterize the two species, they do offer a possible means by which these organisms can be subgrouped.

An antigenic relationship between $C$. chauvoei and C. septicum was described by Moussa (1959), based on somatic, flagellar and spore agglutination antigens. $C$. chavvoei and $C$. septicum share a common spore antigen, but they possess distinguishable somatic and flagellar antigens. These results were supported by those of Batty \& Walker (1963) in their study using a fluorescent antibody technique. In fact, we also demonstrated that there were no cross-reactions in somatic agglutination, using antisera raised against formalin-treated cells, between the $C$. chauvoei and C. septicum strains included in this study (data not shown). Moussa (1959) claimed that there was no reason to regard $C$. chauvoei and $C$. septicum as two distinct species. Although our results define antigenic similarities of limited soluble antigens only, they provide corroborative evidence for the close relationship between $C$. chauvoei and $C$. septicum to the extent that these organisms could be regarded as a single species.

No attempt was made in this study to define the serological relation between $C$. chauvoei and $C$. perfringens which was previously reported by some workers (Poxton \& Byrne, 1984; Reiche \& Bohm, 1991).

We thank Mr M. Wada for technical assistance.

\section{References}

Batty, I. \& Walker, P. D. (1963). The differentiation of Clostridium septicum and Clostridium chauvoei by the use of fluorescent labelled antibodies. Journal of Pathology and Bacteriology 85, 517-521.

Cato, E. P., Hash, D. E., Holdeman, L. V. \& Moore, W. E. C. (1982). Electrophoretic study of Clostridium species. Journal of Clinical Microbiology 15, 688-702.

Cato, E. P., George, W. L. \& Finegold, S. M. (1986). List of species of the genus Clostridium; C. chauvoei, C. septicum. In Bergey's Manual of Systematic Bacteriology, vol. 2, pp. 1163, 1188-1189. Edited by P. H. A. Sneath and others. Baltimore: Williams \& Wilkins.

LAEMmLI, U. K. (1970). Cleavage of structural proteins during the assembly of the head of bacteriophage T4. Nature, London 227, 680-685.

Moussa, R. S. (1959). Antigenic formulae for Clostridium septicum and Clostridium chauvoei. Journal of Pathology and Bacteriology $\mathbf{7 7}$, 341-350.

NaKamura, S., OKado, I., AbE, T. \& Nishida, S. (1979). Taxonomy of Clostridium tetani and related species. Journal of General Microbiology 113, 29-35.

Poxton, I. R. (1984). Demonstration of the common antigens of Clostridium botulinum, $C$. sporogenes and $C$. novyi by enzyme-linked immunosorbent assay and electroblot transfer. Journal of General Microbiology 130, 975-981.

Poxton, I. R. \& BYRNE, M. D. (1981). Immunological analysis of the EDTA-soluble antigens of Clostridium difficile and related species. Journal of General Microbiology 122, 41-46.

Poxton, I. R. \& Byrne, M. D. (1984). Demonstration of shared antigens in the genus Clostridium by an enzyme-linked immunosorbent assay. Journal of Medical Microbiology 17, 171-176.

ReICHE, TH. \& BoHM, R. (1991). Untersuchungen über die Möglichkeit zur serologischen Differenzierung von Clostridium species mittels Enzym-Immun-Verfahren. Journal of Veterinary Medicine, Ser. B. 38, 269-282.

Smith, L. D. S. \& Williams, B. L. (1984). Clostridium chauvoei. In The Pathogenic Anaerobic Bacteria, pp. 164-175. Edited by A. Balows, Illinois: Charles C. Thomas.

Towbin, H., Staehelin, T. \& GoRdon, J. (1979). Electrophoretic transfer of proteins from polyacrylamide gels to nitrocellulose sheets: procedure and some applications. Proceedings of the National Academy of Sciences of the United States of America 76, 4350-4354.

WeinberG, M. \& Mihailesco, M. (1929). Recherches sur le charbon symptomatique et le B. chauvoei. Annales de l'Institut Pasteur 43, 1408-1464.

Willis, A. T. (1969). Clostridium chauvoei and Clostridium septicum. In Clostridia of Wound Infection, pp. 189-208. London: Butterworths. 